\title{
IncRNA HOTAIRM1 regulates cell proliferation and the metastasis of thyroid cancer by targeting Wnt10b
}

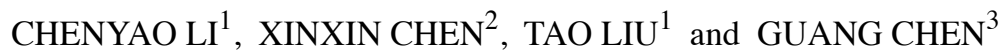 \\ Departments of ${ }^{1}$ Colorectal and Anal Surgery, ${ }^{2}$ Burn Surgery and ${ }^{3}$ Thyroid Surgery, \\ The First Hospital of Jilin University, Changchun, Jilin 130021, P.R. China
}

Received February 28, 2020; Accepted November 11, 2020

DOI: $10.3892 /$ or.2020.7919

\begin{abstract}
Long non-coding RNAs play a role in a variety of malignancies, such as thyroid cancer (TC). However, the effects and function of lincRNA HOTAIRM1 (LINC HOTAIRM1) in TC remains obscure. In the present study, the expression of HOTAIRM1 was evaluated in TC tissues and cells by RT-qPCR and the association between the lncRNA and disease progression was assessed. In vitro, the biological function of HOTAIRM1 was assessed in TC. Moreover, changes in the expression of Wnt10b were measured by western blot analysis. In addition, MTT assay, bioinformatics analysis and luciferase assays were performed to determine the target binding effect between LINC HOTAIRM1 and miR-148a, as well as that between Wnt10b and miR-148a. The changes in the metastatic ability of TPC-1 and BCPAP cells were evaluated by Transwell assay. The pronounced upregulated expression of HOTAIRM1 was evident in TC cells and tissues, and was associated with TNM stage and lymph node metastasis. When HOTAIRM1 was knocked down, this inhibited the proliferative and invasive abilities of TPC- 1 and BCPAP cells in vitro. The knockdown of this IncRNA also increased the expression of microRNA-148a (miR-148a) and decreased Wnt10b expression in these cells, whereas transfection with miR-148a inhibitor was sufficient to overcome this Wnt10b downregulation. In line with these results, the overexpression of miR-148a markedly suppressed Wnt10b expression, whereas miR-148a inhibition resulted in the opposite effects. The overexpression of Wnt10b was also sufficient to overcome the effects of miR-148a mimics on TPC-1 and BCPAP cells. Taken together, these results suggest that miR-148a and Wnt10b are downstream effectors of the HOTAIRM1 signaling pathway in TC. This HOTAIRM1/miR-148a/Wnt10 axis may thus be
\end{abstract}

Correspondence to: Dr Guang Chen, Department of Thyroid Surgery, The First Hospital of Jilin University, 71 Xinmin Street, Changchun, Jilin 130021, P.R. China

E-mail: cguang@jlu.edu.cn

Key words: papillary thyroid cancer, IncRNA HOTAIRM1, miR-148a, Wnt10b amenable to therapeutic targeting in order to improve disease outcomes in patients with TC.

\section{Introduction}

Thyroid cancer (TC), is the most common endocrine malignancy (1), with papillary TC accounting for $80-90 \%$ of all TC cases (2). While the 5-year survival rate of the patients with $\mathrm{TC}$ is $>95 \%$, the local invasion or distant metastases of these tumors can result in poor outcomes, as these tumors generally respond poorly to standard treatments $(3,4)$. It is thus essential that the mechanistic basis for TC onset and progression be better understood in an effort to develop reliable approaches for the treatment of patients suffering from these tumors.

HOX antisense intergenic RNA myeloid 1 (HOTAIRM1) is a type of IncRNA, which has reported to be associated with tumor metastasis. HOTAIRM1 may function as regulator of gene expression, which is expressed from HOXA genomic cluster between HOXA1 and HOXA2. For example, HOTAIRM1 has been reported to exert a crucial effect on multiply types of cancer, such as breast (5), colorectal cancer (6) and glioma (7). However, it remains unclear whether HOTAIRM1 contributes to the malignant progression of TC. To explore the effect and biology function of HOTAIRM1 in $\mathrm{TC}$, the present study assessed its expression, biological functions and the underlying molecular pathways in TC cells.

MicroRNAs (miRNAs or miRs, 18-22 nucleotides in length) are endogenous short noncoding single-stranded RNAs, which have been been reported to induce messenger RNA (mRNA) degradation or block translation by interacting with the 3'-untranslated region (UTR) of target mRNAs $(8,9)$. miRNAs have been reported regulate cellular physiological processes via complex mechanisms. Multiple studies have demonstrated that miR-148a is involved in cervical (10), pancreatic (11), colorectal (12) and gastric cancer (13). However, to date, there is no evidence of an association between HOTAIRM1 and miR-148a in TC, at least to the best of our knowledge.

Herein, it was found that the long non-coding RNA (lncRNA) HOTAIRM1 was significantly upregulated in TC tumor tissues and cells. The knockdown of this lncRNA in TC cell lines was sufficient to impair their proliferative and invasive activity, while simultaneously promoting the upregulation of miR-148a and the downregulation of Wnt10b. The inhibition of miR-148a was sufficient to reverse this reduction in Wnt10b 
expression, and miR-148 overexpression exerted the opposite effect, owing to the ability of this miRNA to directly bind to the Wnt10b 3'-UTR. The overexpression of Wnt10b reversed the effect of miR-148a mimics on both TPC-1 and BCPAP cells. Taken together, the data thus suggest that HOTAIRM1 knockdown can suppress the proliferation and metastasis of TC cells via modulating the miR-148a/Wnt10b axis.

\section{Materials and methods}

Tissue specimens. A total of 52 pairs clinical tissues were obtained from patients with TC at the First Hospital of Jilin University from March 2019 to November 2019. Written informed consent was obtained from all study subjects. The present study conformed to the principles presented in the Declaration of Helsinki and was approved (no. 20190318) by the Ethics Committee of the First Hospital of Jilin University (Jilin, China).

Cells and cell culture. Human TPC-1 [cat. no. CC-Y1522; Meiyan (Shanghai) Biological Technology Co., Ltd.], BCPAP [cat. no. CC-Y1064; Meiyan (Shanghai) Biological Technology Co., Ltd.] and Nthy-ori 3-1 [cat. no. CC-Y1708; Meiyan (Shanghai) Biological Technology Co., Ltd.] cells were grown in DMEM supplemented $10 \%$ fetal bovine serum (FBS; Thermo Fisher Scientific, Inc.) at $37^{\circ} \mathrm{C}$ in a $5 \% \mathrm{CO}_{2}$ incubator.

Construction of lentiviruses. The primer sequences targeting IncRNA HOTAIRM1 (Table I) were chemically synthesized, and each was inserted into the AgeI-EcoRI site of the pLKO.1-Puro vector purchased from Beijing Solarbio Science \& Technology Co., Ltd. The coding sequence (CDS) of Wnt10b was synthesized from GenePharma Co., Ltd., and inserted into the EcoRI-BamHI site of the pLVX-Puro vector (Shanghai Yubo Biotechnology Co., Ltd.). Subsequently, HOTAIRM1-specific shRNAs (GenePharma Co., Ltd.) or pLKO.1-Puro vector of the lentiviral particles were co-transfected (1:1.5) into 293T cells (ATCC) with the mixed set of packaging plasmids (SPAX2 and MD2G) using Lipofectamine 3000 (Invitrogen; Thermo Fisher Scientific, Inc.). Following $48 \mathrm{~h}$ of incubation at $37^{\circ} \mathrm{C}$, viral particles were achieved by ultracentrifugation $(12,000 \mathrm{x}$ g for $1 \mathrm{~min}$ at room temperature).

Cell treatment. TPC-1 and BCPAP cells were transduced with lentiviruses encoding HOTARIRM1-specific shRNAs (shHOTAIRM1-1 and shHOTAIRM1-2), corresponding negative controls (shNC), or a Wnt10b overexpression (Wnt10b) vector (all from RiboBio Co. Ltd.) at 1:1.2, after which reverse transcription-quantitative PCR (RT-qPCR) was used to determine the efficacy of HOTAIRM1 knockdown or Wnt10b overexpression.

Following lentiviral transfection with shHOTAIRM1-1 (5'-AGAAACUCCGUGUUACUCAUU-3'), shHOTAIRM1-2 (5'-GCCAGAAACCAGCCAUAGU-3') or shNC, MTT and Transwell assays were respectively used to determine the proliferation and invasion of the transduced cells. Furthermore, the expression of Wnt10b and specific miRNAs in these cells was analyzed. miR-148a mRNA, Wnt10b expression and luciferase activity were assessed following transfection with miR-148a mimic or inhibitor at 1:1.5 at room temperature.
For individual experiments, cells were transfected with lentiviral constructs and miR-148a inhibitors/mimic and respective negative controls (Guangzhou RiboBio Co. Ltd.) using DharmaFECT 1 (Qcbio Science \& Technologies Co., Ltd.) transfection reagent at 1:1.5 with the following combinations: shNC, shNC + miR-148a inhibitor, shHOTAIRM1, shHOTAIRM1 + miR-148a inhibitor. Moreover, cells were stimulated with miR-148a mimics, miR-148a mimics + Wnt10b, NC + Wnt10b, or NC alone. Following the indicated treatments, cellular proliferation, invasion and gene expression were analyzed.

$R T$ - $q P C R$. Total RNA was extracted from the TC cells and tissues using TRIzol reagent (Invitrogen; Thermo Fisher Scientific, Inc.). A total of $1 \mu \mathrm{g}$ RNA was reverse transcribed into cDNA using the PrimeScript ${ }^{\mathrm{TM}} \mathrm{RT}$ reagent kit (Takara Biotechnology Co., Ltd.). RT-qPCR was performed using the CFX96 system using SYBR ${ }^{\circledR}$ Premix Ex Taq ${ }^{\mathrm{TM}}$ II kit (Takara Biotechnology Co., Ltd.). The thermocycling conditions were as follows: $95^{\circ} \mathrm{C}$ for $30 \mathrm{sec}, 95^{\circ} \mathrm{C}$ for $10 \mathrm{sec}$, $60^{\circ} \mathrm{C}$ for $30 \mathrm{sec}, 35$ cycles. GAPDH was used as the endogenous control. The $\mathrm{Cq}$ values was calculated through the $2^{-\Delta \Delta \mathrm{Cq}}$ method (14).

Likewise, miRNAs were reverse transcribed and miRNA-specific primers $(10 \mu \mathrm{M})$ (Table II). miRNA expression was detected using the TaqMan microRNA assay kit (Applied Biosystems; Thermo Fisher Scientific, Inc.), with an ABI7900 real-time PCR system (Applied Biosystems; Thermo Fisher Scientific, Inc.). The thermocycling conditions were as follows: $95^{\circ} \mathrm{C}$ for $5 \mathrm{~min}, 95^{\circ} \mathrm{C}$ for $30 \mathrm{sec}, 58^{\circ} \mathrm{C}$ for $20 \mathrm{sec}$, 40 cycles. U6 was used as the endogenous control.

Western blot analysis. Total protein was extracted from the treated and untreated TC cells using RIPA buffer (Beijing Solarbio Science \& Technology Co., Ltd.). Following quantification using a BCA quantification kit (Beijing Solarbio Science \& Technology Co., Ltd.), $50 \mu \mathrm{g}$ protein were separated in a $10 \%$ SDS-PAGE gels and transferred onto a polyvinylidene fluoride (PVDF) membranes (EMD Millipore). The membranes were blocked for $1 \mathrm{~h}$ at room temperature with $5 \%$ no-fat milk (BD Biosciences), followed by incubation with the primary antibodies overnight at $4{ }^{\circ} \mathrm{C}$. The primary antibodies used were as follows: Against Wnt10b $(1: 1,000$; ab70816; Abcam) and GAPDH (1:2,000; ab9485; Abcam). The following day, after 3 washes with $0.1 \%$ TBST at room temperature, the membranes were incubated with HRP-conjugated goat anti-rabbit $\operatorname{IgG}(1: 2,000 ;$ ab6721; Abcam) for $2 \mathrm{~h}$ at room temperature. Following 3 washes with $0.1 \%$ TBST, the blots were incubated for $3 \mathrm{~min}$ at room temperature with ECL reagent (EMD Millipore) and exposed on an imaging system (Tanon Science \& Technology Co., Ltd.). Protein density values were assessed and calculated using ImageJ software (version 1.47; National Institutes of Health).

3-(4,5-Dimethylthiazol-2-yl)-2,5-diphenyltetrazolium bromide assay. A 3-(4,5-dimethylthiazol-2-yl)-2,5-diphenyltetrazolium bromide (MTT) assay was used to assess TC cell proliferation following the indicated treatments. Briefly, at $0,24,48$ or $72 \mathrm{~h}$ post-treatment, the cells were treated with MTT $(5 \mathrm{mg} / \mathrm{ml})$ for $4 \mathrm{~h}$. Dimethyl sulfoxide was then used to dissolve the 
Table I. shRNA sequences used for HOTAIRM1.

\begin{tabular}{lc}
\hline shRNA name & Sequence $\left(5^{\prime}-3^{\prime}\right)$ \\
\hline shHOTAIRM1-1 & CCGGGCCTCTATTACCAATTTAAATTCTCGAGTTAAATTGGTAATAGAGGCAGTTTTTTG \\
shHOTAIRM1-2 & AATTCAAAAAACTGCCTCTATTACCAATTTAACTCGAGATTTAAATTGGTAATAGAGGC
\end{tabular}

Table II. Primers for the target gene and primers for Wnt10b 3'UTR used for RT-qPCR and in the luciferase report assay.

\begin{tabular}{ll}
\hline Gene & \multicolumn{1}{c}{ Sequence $\left(5^{\prime}-3^{\prime}\right)$} \\
\hline miR-148a & F: GGCAGCAAAGTTCTGAGACAC \\
RiR-141-3p & F: ACACTCCAGCTGGGCATCTTCCAG \\
& R: CTCAACTGGTGTCGTGGAGTCGGCAATTCAGTTGAGTCCAAC \\
miR-1 & F: CAGTGCGTGTCGTGGAGT \\
& R: GGCCTGGAATGTAAAGAAGT \\
miR-21 & F: GCACCGTCAAGGCTGAGAAC \\
& R: CAGCCCATCGACTGGTG \\
Wnt 10b & F: TGGAAGAATGCGGCTCTGAC \\
& R: AGAGTGACCTTGGAAGGAAATC \\
GAPDH & F: TCACCA GGGCTGCTTTTAAC \\
U6 & R: TGACGGTGCCA TGGAATTTG \\
& F: CTCGCTTCGGCAGCACA \\
Wt 3'UTR of Wnt10b & R: AACGCTTCACGAATTTGCGT \\
& F: CTAGCTAGCGGCCGCTAGTTGGACTAAGATG AAATGCACTGTG \\
Mut 3'UTR of Wnt10b & R: TCGACACAGTGCATTTCATCTTAGTCCAACTA GCGGCCGCTAG \\
& F: CTAGCTAGCGGCCGCTAGTTGGACTAAGATG AAAAGGAGTCTG \\
& R: TCGACAGACTCCTTTCATCTTAGTCCAACTA GCGGCCGCTAG
\end{tabular}

F, forward; R, reverse; 3'-UTR, 3'-untranslated region.

resultant formazan crystals, and the absorbance (OD) values at $570 \mathrm{~nm}$ were measured using a plate reader (ELX800; Biotek Instruments, Inc.).

Transwell invasion assay. The upper chamber of a Transwell plate insert was coated with Matrigel (BD Biosciences). Cells $\left(5 \times 10^{4}\right)$ appropriately treated in serum-free medium were added to the upper chamber at $24 \mathrm{~h}$ post-treatment, while medium containing $10 \%$ FBS was added to the lower chamber. Following $48 \mathrm{~h}$ of incubation at $37^{\circ} \mathrm{C}$, cells that remained in the upper chamber were carefully removed using cotton swab, while $4 \%$ paraformaldehyde was used to stain the remaining cells for $10 \mathrm{~min}$ at room temperature, and invasive cells in 6 random fields of view per samples were counted using a light microscope (Olympus Corporation).

Luciferase reporter assay. The cells $\left(5 \times 10^{5}\right.$ per well) were added to 6 -well plates for $24 \mathrm{~h}$ at $37^{\circ} \mathrm{C}$, after which they were transfected with $1.5 \mu \mathrm{g}$ of a luciferase plasmid (pGL3-promoter Wnt10b) and $5 \mu \mathrm{l}$ of NC miRNA (5'-CAGUACUUUUGU GUAGUACAA-3'), $1.5 \mu \mathrm{g}$ of luciferase plasmid and $5 \mu \mathrm{l}$ of miR-148a inhibitor, or $1.5 \mu \mathrm{g}$ luciferase plasmid and $5 \mu \mathrm{lmiR}-148 \mathrm{a}$ mimics for $6 \mathrm{~h}$ at $37^{\circ} \mathrm{C}$ using Lipofectamine
3000 (Invitrogen; Thermo Fisher Scientific, Inc.). At 48 h post-transfection, a Dual-Luciferase Reporter Assay System (Promega Corporation) was used to assess the luciferase activity in these cells, with Renilla luciferase activity being used for normalization.

Targeting of 148a. TargetScan (http://www.targetscan.org) software program was used to search for miR-148a target genes.

Statistical analysis. SPSS 22.0 software (IBM, Inc.) was used for all statistical analyses. All data are the means \pm the standard deviation (SD), and were compared using Student's t-tests or one-way analysis of variance (ANOVA) with the Tukey's post hoc test. The Kaplan-Meier analysis and the log rank test were used for the survival analysis. HOTAIRM1 expression was analyzed using Fisher's exact probability test in Excel. A value of $\mathrm{P}<0.05$ was considered to indicate a statistically significant difference.

\section{Results}

PTC tissues and cells exhibit HOTAIRM1 upregulation. The present study began by comparing the expression of 
Table III. Association between clinicopathological features and HOTAIRM1 expression in 52 patients with thyroid carcinoma.

HOTAIRM1 expression

\begin{tabular}{|c|c|c|c|c|}
\hline & & & & \\
\hline Variables & No. of patients & High (\%) & Low $(\%)$ & $\mathrm{P}$-value \\
\hline Age (years) & & & & 0.976 \\
\hline$<60$ & 23 & $12(52.17)$ & $11(47.83)$ & \\
\hline$\geq 60$ & 29 & $13(44.83)$ & $16(55.17)$ & \\
\hline Sex & & & & 0.373 \\
\hline Male & 17 & $5(29.41)$ & $12(70.59)$ & \\
\hline Female & 35 & $20(57.14)$ & $15(42.86)$ & \\
\hline TNM stage & & & & 0.018 \\
\hline I-II & 18 & $4(22.22)$ & $14(77.78)$ & \\
\hline III-IV & 34 & $21(61.67)$ & $13(38.23)$ & \\
\hline Tumor size & & & & 0.427 \\
\hline$<1 \mathrm{~cm}$ & 21 & $9(42.86)$ & $12(57.14)$ & \\
\hline$\geq 1 \mathrm{~cm}$ & 31 & $16(51.61)$ & $15(48.39)$ & \\
\hline Lymph node metastasis & & & & 0.014 \\
\hline Yes & 33 & $22(66.67)$ & $11(33.33)$ & \\
\hline No & 19 & $3(15.79)$ & $16(84.21)$ & \\
\hline
\end{tabular}

A

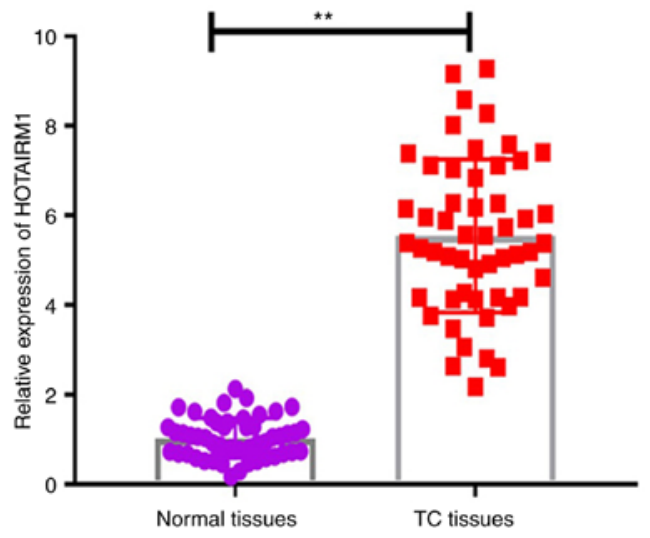

B
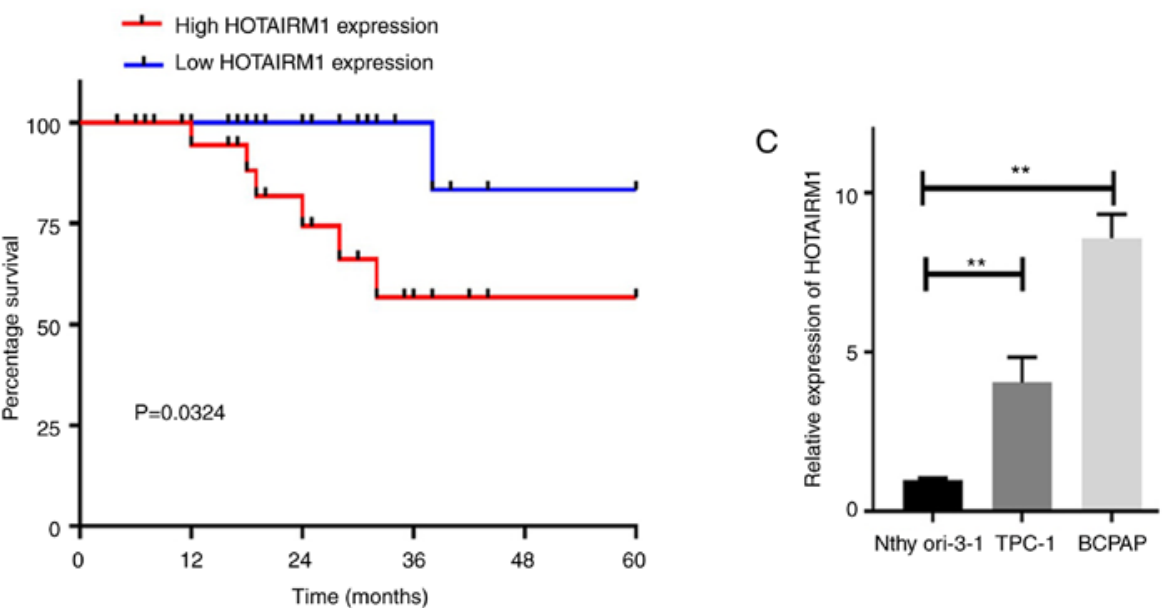

Figure 1. TC tissues and cells exhibit an upregulated HOTAIRM1 expression. (A) RT-qPCR was used to assess HOTAIRM1 expression in human TC and paracancerous tissue samples $(\mathrm{n}=52)$. (B) TC patient survival curves for individuals exhibiting low or high levels of HOTAIRM1 expression ( $\mathrm{n}=52$ ). (C) HOTAIRM1 levels were measured in TPC-1 and BCPAP PTC cell lines and in the Nthy-ori $3-1$ control cell line ( $\mathrm{n}=4$ repeats). ${ }^{* *} \mathrm{P}<0.01$. TC, thyroid cancer.

HOTAIRM1 in human TC tumor and paracancerous tissue samples, revealing that this lncRNA was markedly upregulated in cancerous tissues (Fig. $1 \mathrm{~A} ; \mathrm{P}<0.01$ ). It was also found that HOTAIRM1 upregulation was closely associated with patient 

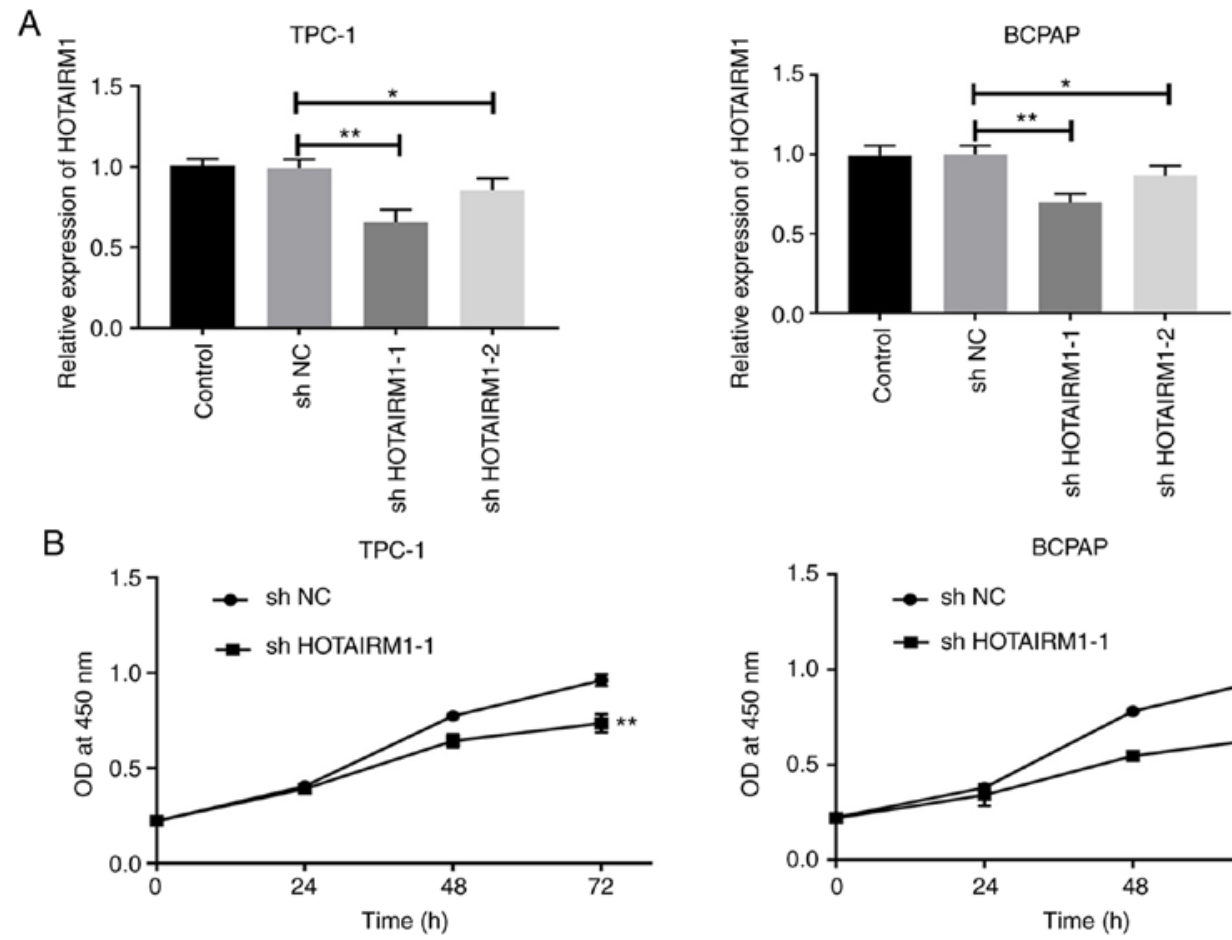

C
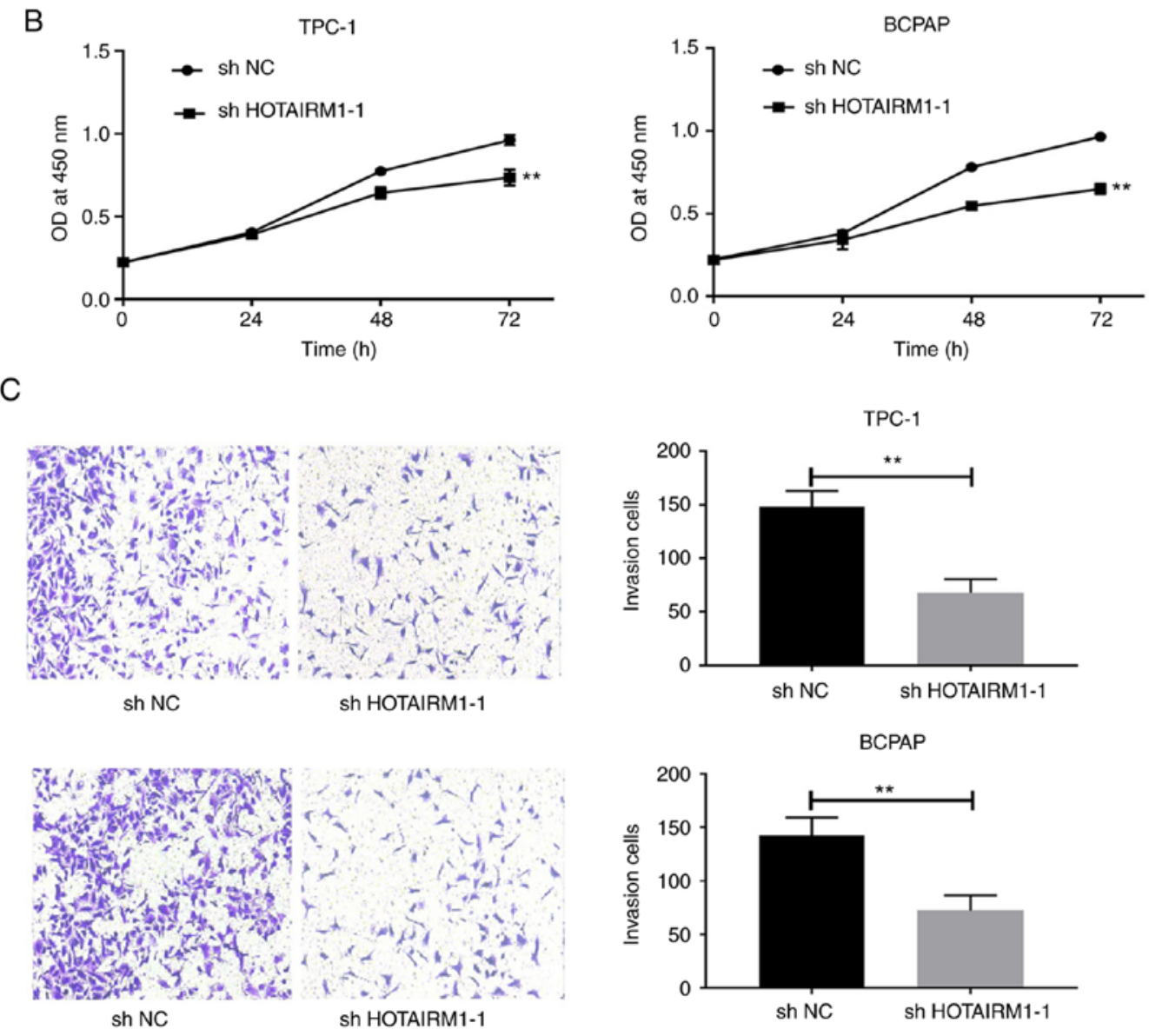

Figure 2. HOTAIRM1 controls the invasion and proliferation of TC cells. (A) Lentivirus-mediated HOTAIRM1 knockdown efficiency was assessed by RT-qPCR ( $\mathrm{n}=4$ repeats). (B) Cell proliferation was measured at $0,24,48$ and $72 \mathrm{~h}$ by MTT assays $(\mathrm{n}=3)$. (C) A Transwell assay was used to evaluate cell invasion following HOTAIRM1 knockdown ( $\mathrm{n}=6$ repeats). ${ }^{*} \mathrm{P}<0.05,{ }^{* *} \mathrm{P}<0.01$. TC, thyroid cancer.

TNM stage and lymph node metastasis, whereas it was not associated with patient age, sex, or tumor size (Table III; $\mathrm{P}<0.05)$. The long-term survival of patients expressing higher HOTAIRM1 levels was also significantly decreased relative to that of patients expressing lower levels of this lncRNA (Fig. 1B; $\mathrm{P}<0.05$ ).

The expression of HOTAIRM1 was then compared in TC and control cell lines, revealing that this lncRNA was expressed at significantly higher levels in the TPC-1 and BCPAP PTC cell lines relative to the control Nthy-ori 3-1 cell line (Fig. 1C; $\mathrm{P}<0.01)$. Thus, the TPC-1 and BCPAP cells were selected for use in further analyses in order to better understand the functional relevance of HOTAIRM1 in TC.
Knockdown of HOTAIRM1 impairs TC cell proliferation and invasion. Subsequently, HOTAIRM1 expression was successfully knocked down in TC cell lines using lentiviruses encoding shHOTAIRM1 (Fig. 2A; P<0.05). The knockdown of this lncRNA significantly impaired TPC-1 and BCPAP cell proliferation (Fig. 2B, $\mathrm{P}<0.05$ ) and invasion (Fig. $2 \mathrm{C}$, $\mathrm{P}<0.05)$. Thus, HOTAIRM1 may drive TC cell proliferation and metastasis.

miR-148a represents a putative HOTAIRM1 downstream target in TC. The miRNA expression patterns in TC cells following the knockdown of HOTAIRM1 were then evaluated. HOTAIRM1 knockdown promoted the upregulation of 
A 色
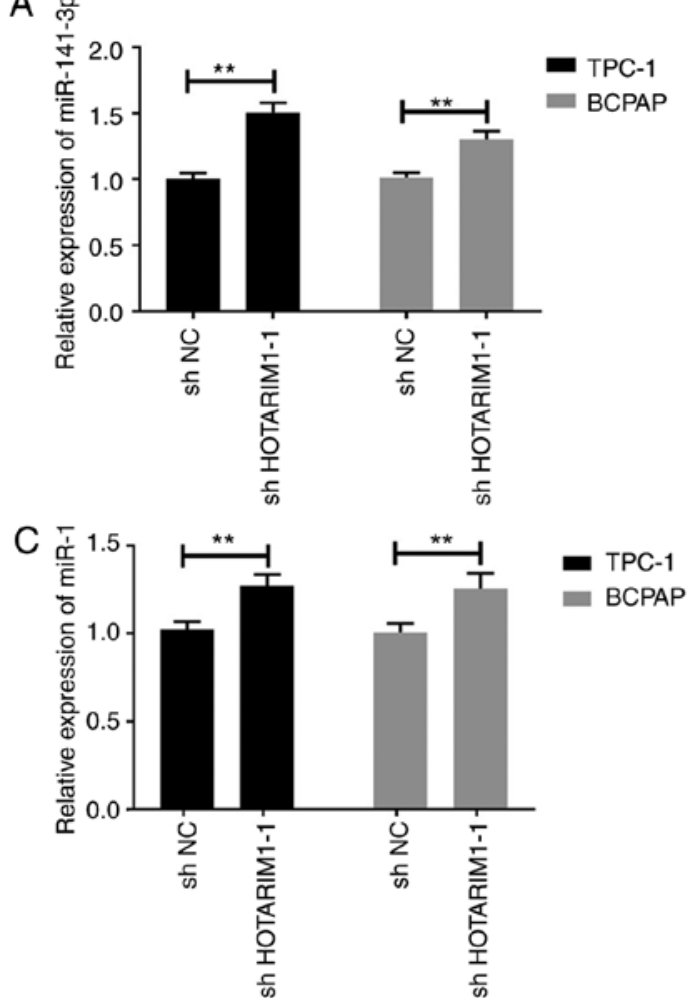

E

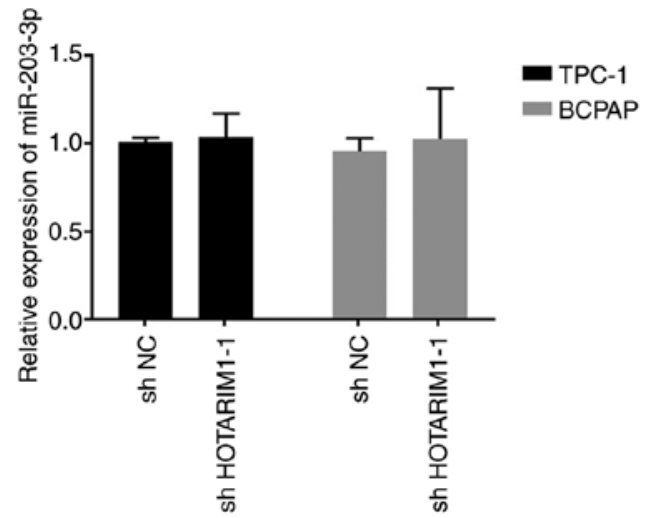

B
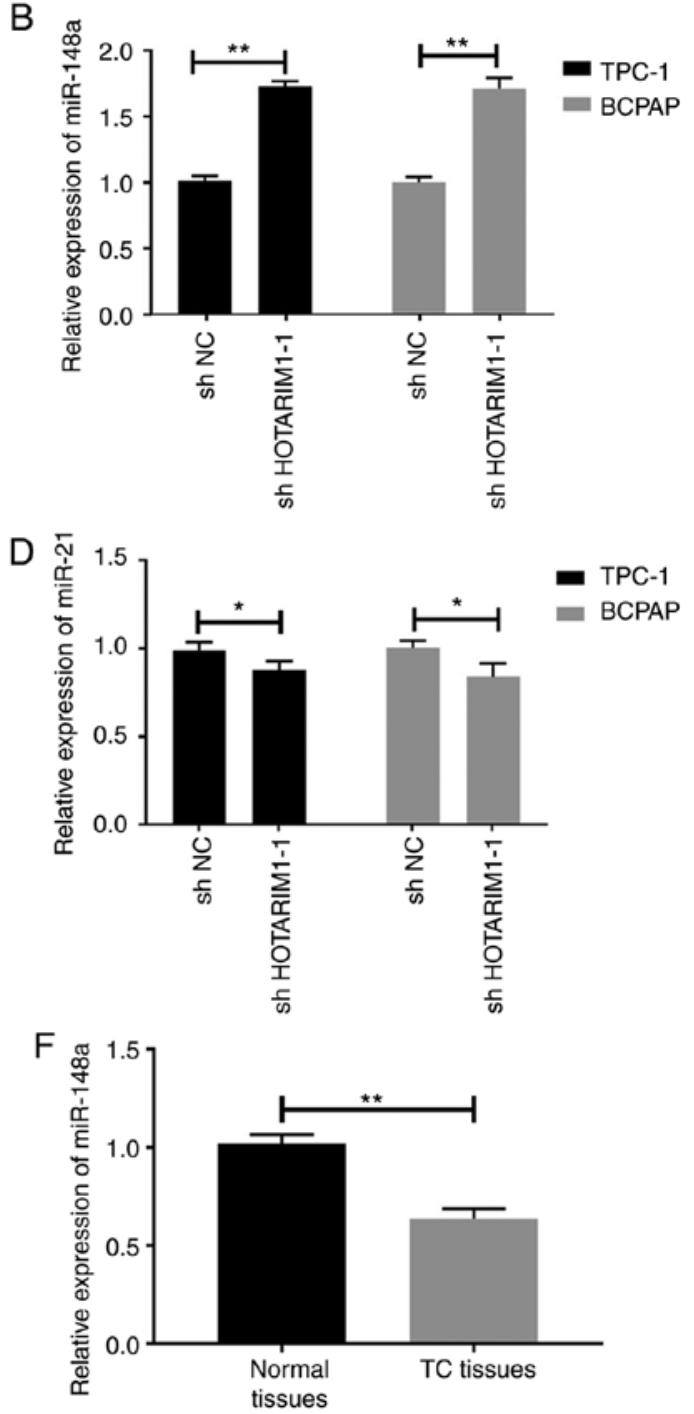

Figure 3. miR-148a is a HOTAIRM1 downstream target in TC. TC cells were transduced with lentiviruses encoding shNC or shHOTAIRM1-1. (A-E) The mRNA expression of (A) miR-141-3p, (B) miR-148a, (C) miR-1, (D) miR-21 and (E) miR-203-3p were determined by RT-qPCR (n=3 repeats). (F) miR-148a mRNA in normal adjacent tissues and PTC tissues was detected by RT-qPCR ( $\mathrm{n}=3$ repeats). ${ }^{*} \mathrm{P}<0.05,{ }^{* *} \mathrm{P}<0.01$. TC, thyroid cancer.

miR-141-3p (Fig. 3A, P<0.05), miR-148a (Fig. 3B, P <0.05) and miR-1 (Fig. 3C, $\mathrm{P}<0.05$ ) in TC cells, whereas it suppressed the expression of miR-21 (Fig. 3D, P<0.05). No marked changes in the expression of miR-202-3p were observed as a function of HOTAIRM1 knockdown (Fig. 3E, P>0.05). Of the miRNAs examined, miR-148a exhibited the most marked changes relative to baseline expression, and it was also found that miR-148a was significantly downregulated in tissues from patients with TC relative to the paracancerous tissue samples (Fig. 3F, $\mathrm{P}<0.01$ ).

Inhibition of miR-148a reverses the effects of HOTAIRMI knockdown on TC cells. The potential association between HOTAIRM1 and miR-148a was then evaluated in TC cells. It was found that transfection with miR-148a inhibitor following HOTAIRM1 knockdown was sufficient to restore TC cell proliferation (Fig. 4A, $\mathrm{P}<0.01$ ) and invasion (Fig. 4B, $\mathrm{P}<0.01$ ), indicating that this lncRNA controls TC cell proliferation and invasion, at least in part by regulating miR-148a.

miR-148a directly suppresses Wnt10b expression in TC cells. Subsequently, the mechanisms whereby miR-148a affects TC cells were evaluated. TargetScan software revealed a putative miR-148a binding site within the Wnt10b 3'-UTR. In line with this prediction, it was found that Wnt10b was significantly upregulated in TC tumor tissues relative to paracancerous control tissues (Fig. 5A, P<0.01). Importantly, the knockdown of HOTAIRM1 decreased the protein expression level of Wnt10b in TPC-1 and BCPAP cells (Fig. 5B, P<0.01), whereas transfection with miR-148a inhibitor reversed this effect (Fig. 5C, $\mathrm{P}<0.01)$. Following miR-148a overexpression or inhibition in TC cell lines (Fig. 5D, $\mathrm{P}<0.01$ ), a luciferase reporter assay was then conducted, which revealed that the overexpression of miR-148a suppressed the luciferase activity and the expression 

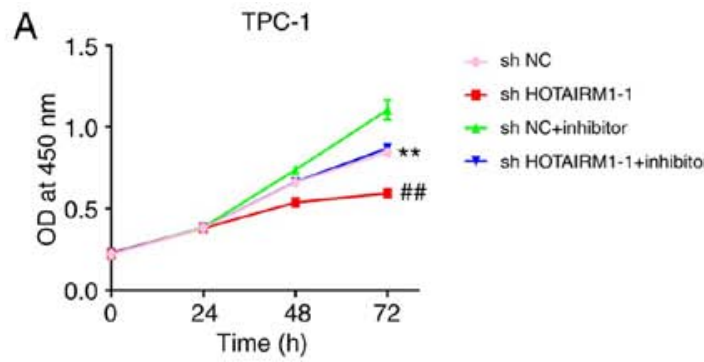

\section{B}
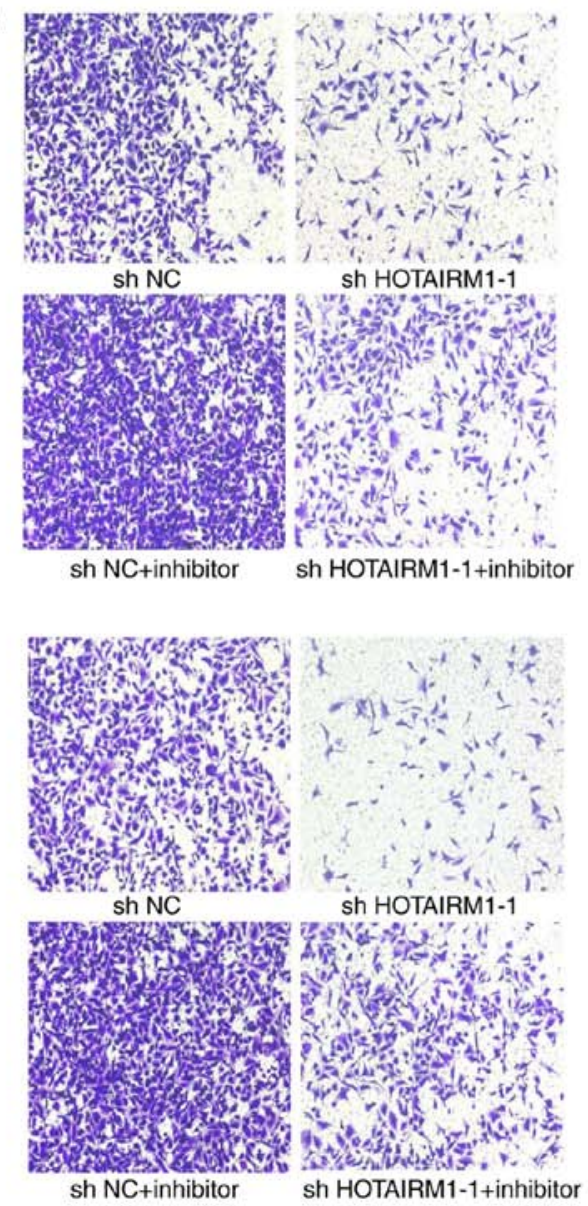
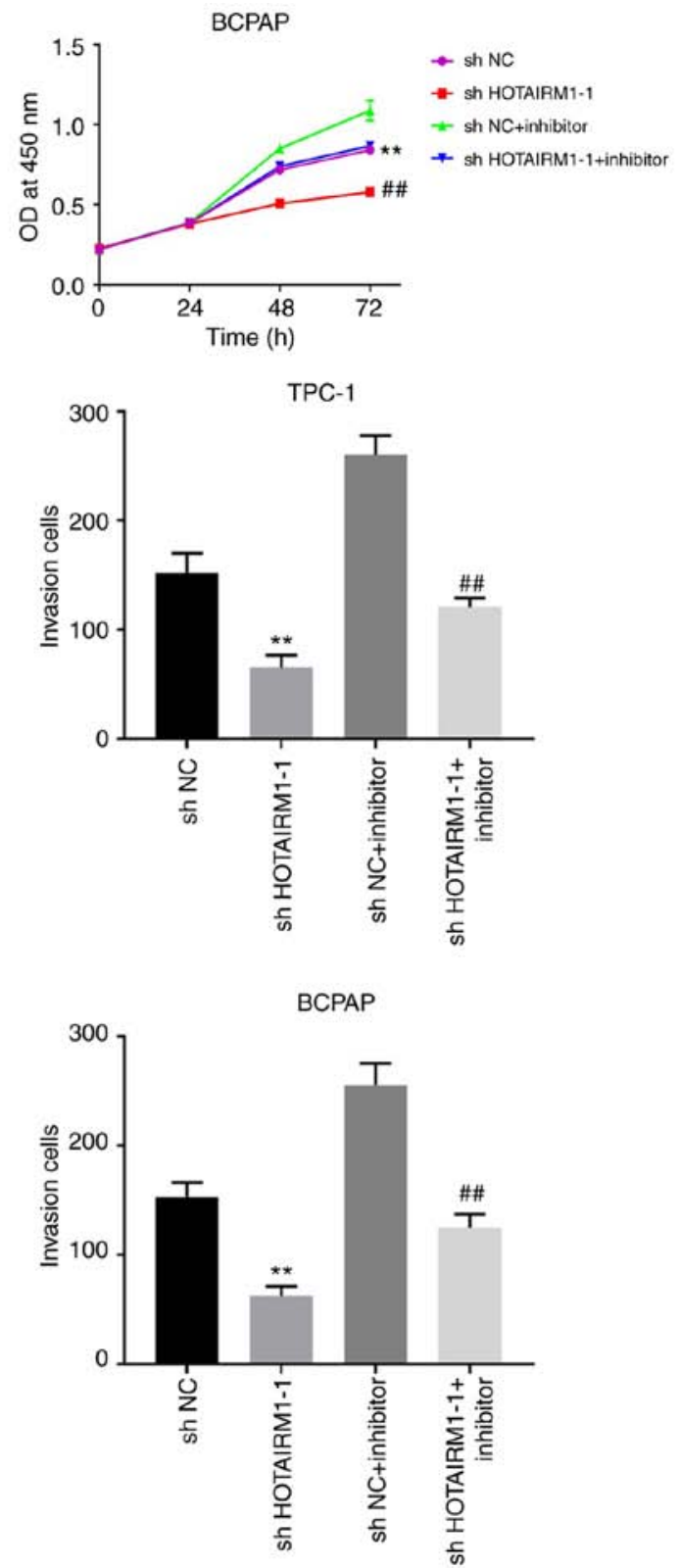

Figure 4. Inhibition of miR-148a reverses the effect of HOTAIRM1 knockdown on TC cells. TC cells were treated with shNC or shHOTAIRM1 \pm miR-148a inhibitor. (A) Cell proliferation was assessed at $0,24,48$ and $72 \mathrm{~h}$ post-treatment by MTT assays ( $\mathrm{n}=3$ repeats). (B) Following treatment, cell invasion was examined by Transwell assay ( $\mathrm{n}=6$ repeats). ${ }^{* *} \mathrm{P}<0.01$ vs. $\mathrm{shNC},{ }^{\# \#} \mathrm{P}<0.01$ vs. shHOTAIRM1. TC, thyroid cancer.

of Wnt10b expression, while miR-148a inhibition resulted in the opposite effect (Fig. 5E-G, P<0.01). Taken together, these findings suggested that miR-148a suppressed Wnt10b expression via binding to the Wnt10b 3'-UTR in TC cells.

miR-148a targets Wnt10b to control TC cell proliferation and invasion. Finally, the functional impact of the overexpression of Wnt10b in TC cells was evaluated using a lentiviral construct (Fig. 6A-C). Following Wnt10b overexpression and simultaneous transfection with miR-148a mimic, it was found that the overexpression of miR-148a was sufficient to impair cell proliferation (Fig. 6C, $\mathrm{P}<0.01$ ) and invasion (Fig. 6D, $\mathrm{P}<0.01$ ), while also suppressing Wnt10b expression (Fig. 6E, $\mathrm{P}<0.01)$ in TPC-1 and BCPAP cells. Wnt10b overexpression, however, was sufficient to reverse these effects induced by miR-148a mimic, thus suggesting that the miR-148a-mediated suppression of Wnt10b expression is an important regulator of TC cell proliferation and invasion.

\section{Discussion}

Herein, it was found that both HOTAIRM1 and Wnt10b were significantly upregulated in TC tissues and cells, whereas miR-148a was downregulated in these samples. The knockdown of HOTAIRM1 and the overexpression miR-148a were both sufficient to suppress the proliferation and invasion of TC cells and to reduce Wnt10b expression in these cells. As such, these data suggested that HOTAIRM1, miR-148a and Wnt10b may serve as key regulators of TC progression.

While the mechanistic basis for TC development remains complex and incompletely understood, several lncRNAs have been shown to regulate key oncogenic processes in 


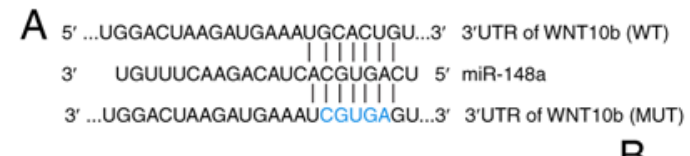

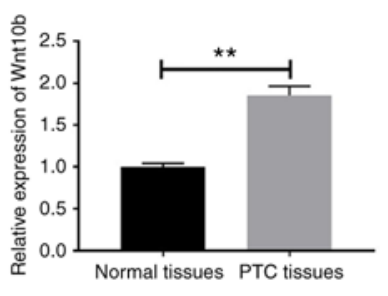

C
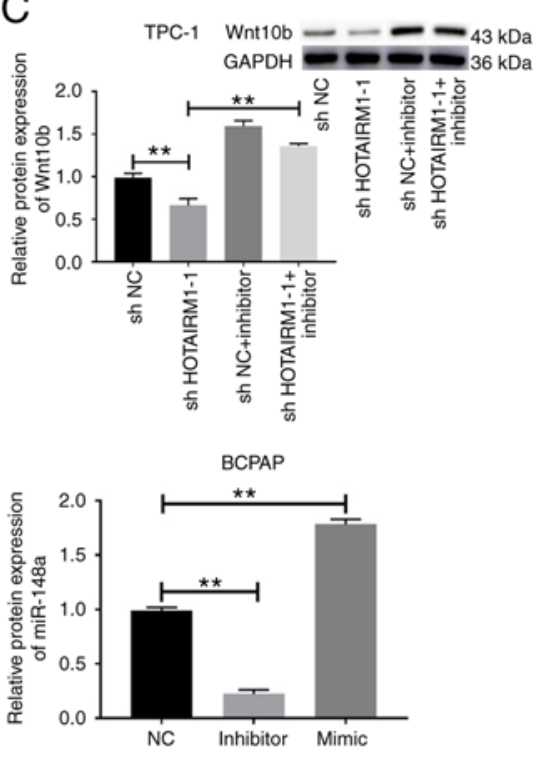

$\mathrm{F}$

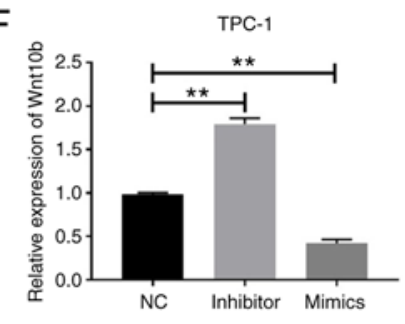

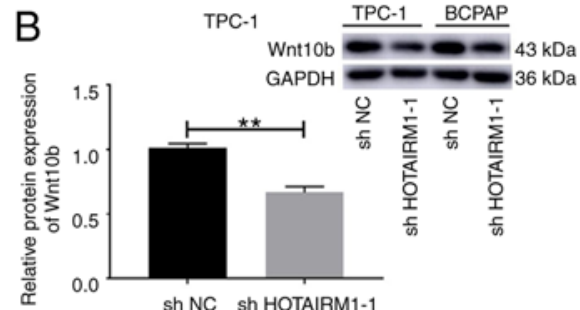

sh NC sh HOTAIRM1-1

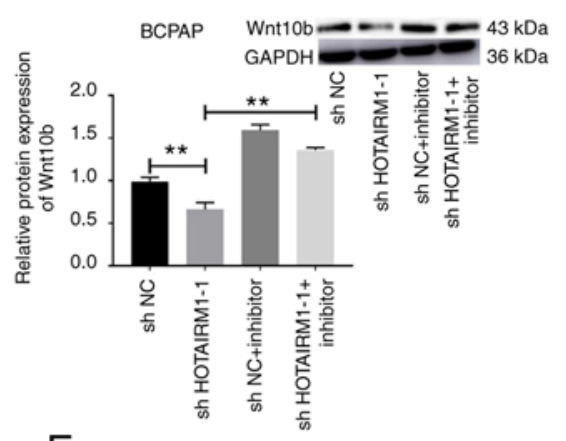

$\mathrm{E}$
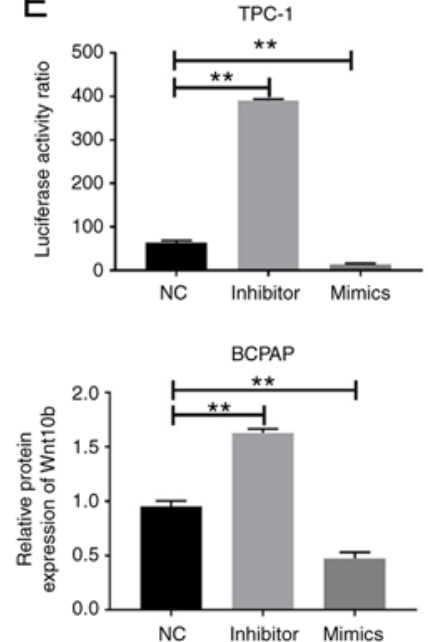

NC Inhibitor Mimics
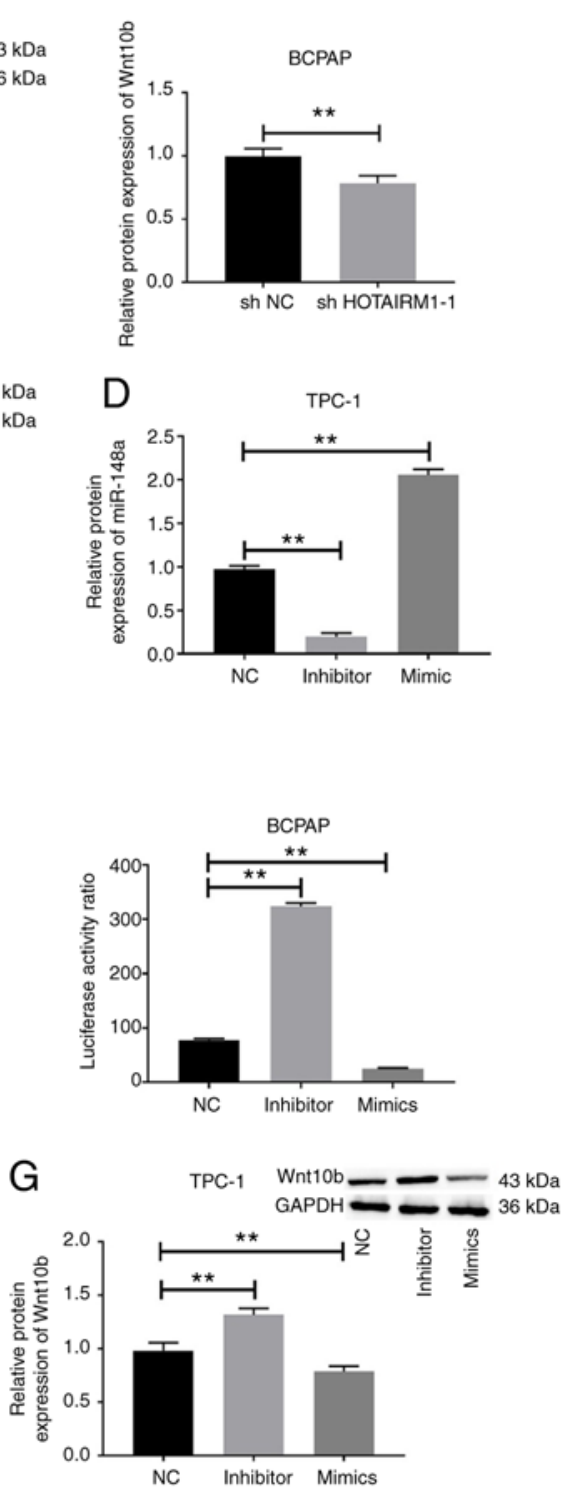

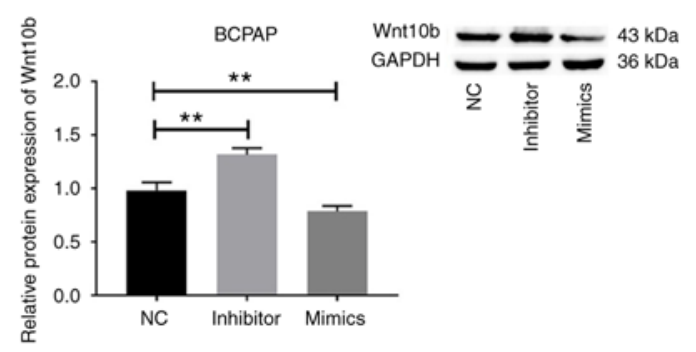

Figure 5. miR-148a directly targets and suppresses Wnt10b expression (A) The expression of Wnt10b in TC was detected by RT-qPCR (n=3). (B and C) Wnt10b protein levels were measured by western blot analysis. Following transfection with NC miRNA, miR-148a inhibitor and miR-148a mimics (n=3). (D) The efficiency of miR-148a inhibitor and mimics on expression was measured by RT-qPCR ( $\mathrm{n}=3$ ). (E) The luciferase activity was detected using a Dual-luciferase assay kit $(\mathrm{n}=3)$. (F and G) Wnt10b mRNA and protein were detected by RT-qPCR and western blot analysis, respectively ( $\mathrm{n}=3$ ). ${ }^{* *} \mathrm{P}<0.01 \mathrm{vs}$. Normal, shNC or NC. TC, thyroid cancer.

this context, including cellular proliferation, migration and epithelial-mesenchymal transition (15-18). As such, the present study sought to evaluate the functional relevance of lncRNAs in TC.

Previous studies have demonstrated that miRNAs can also regulate TC pathogenesis (19-21). For example, miR-141-3p suppresses the growth and metastasis of TC cells (22), while miR-1 serves as a tumor suppressor that constrains the migration and proliferation of TC cells (23). Similarly, miR-148a impairs TC cell proliferation, migration and invasion $(24,25)$, while miR-21 and miR-202-3p also serve as regulators of this cancer type $(26,27)$. 

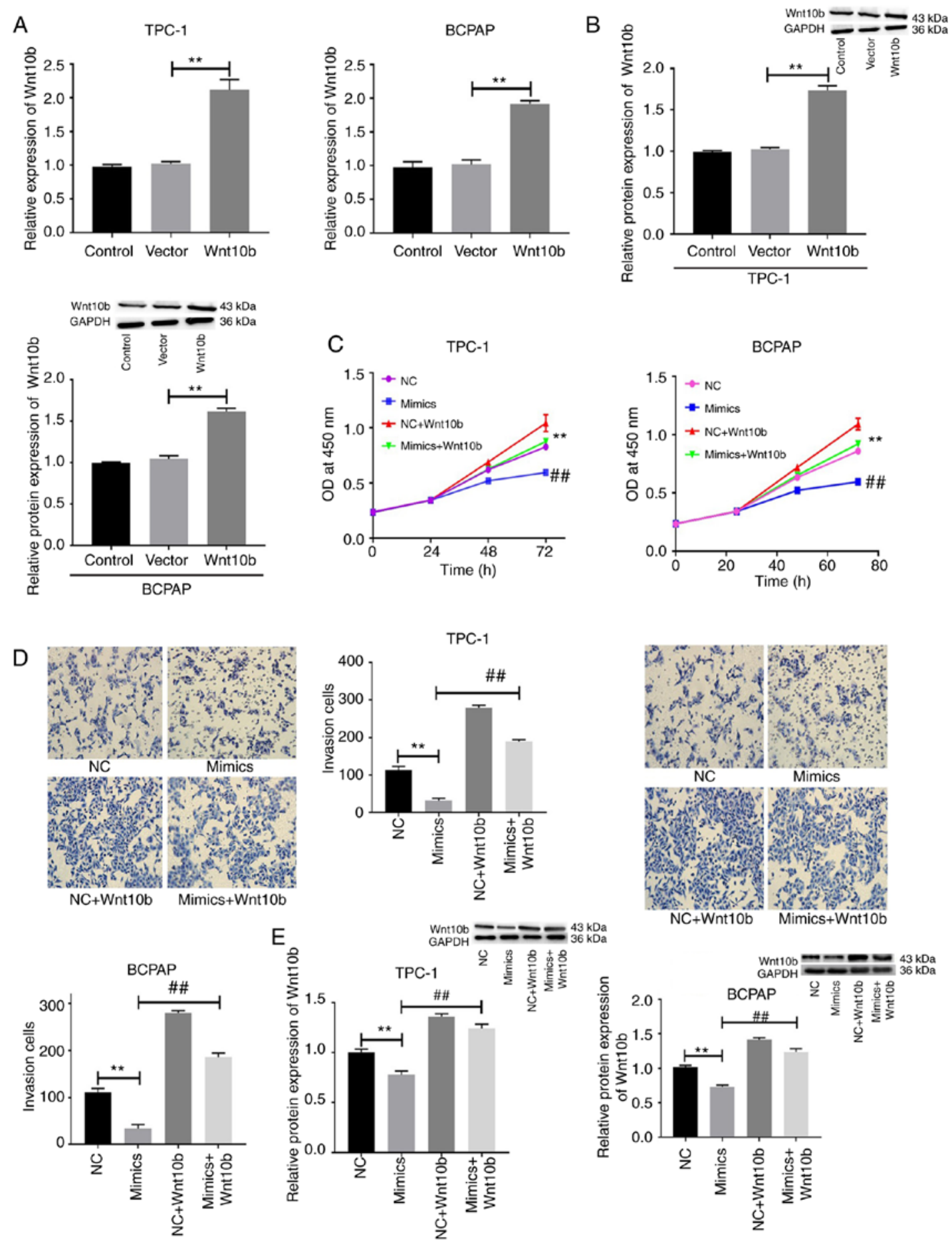

Figure 6. miR-148a targets Wnt10b to regulate TC cell proliferation and invasion. (A and B) The overexpression efficiency of Wnt10b lentivirus in TPC-1 and BCPAP cells was measured by RT-qPCR and western blot analysis $(n=3)$. TPC-1 and BCPAP cells were then transfected with NC miRNA \pm Wnt10b or miR-148a mimics \pm Wnt10b. (C) Cell proliferation following treatment was assessed at $0,24,48$ and $72 \mathrm{~h}$ by MTT assays (n=3). (D) Following treatment, cell invasion was examined by Transwell assay $(n=6)$. (E) Wnt10b protein levels were measured by western blot analysis $(n=3)$. ${ }^{* *} \mathrm{P}<0.01 \mathrm{vs}$. vector or $\mathrm{NC}$, ${ }^{\# \#} \mathrm{P}<0.01$ vs. mimics. TC, thyroid cancer.

Based on the above-mentioned reports, the present study observed that several miRNAs exert marked effects on TC, particularly miR-148a. Furthermore, miR-148a expression in TC cells was negatively regulated by HOTAIRM1, which is consistent with the findings of previous studies $(28,29)$. From these results, it was hypothesized that miR-148a functions downstream of HOTAIRM1. Furthermore, the inhibitory effects of HOTAIRM1 knockdown on the proliferation and invasion of TC cells were potently counteracted by the inhibition of miR-148a. These findings indicate that the HOTAIRM1-regulated cell proliferative and invasive ability in TC is likely modulated by miR-148a.

It was found that miR-148a upregulation in TC cells directly suppressed Wnt10b expression by binding to the Wnt10b 3'-UTR. As such, Wnt10b is a miR-148a target gene that may be linked to TC pathogenesis. Previous studies have demonstrated that a 
range of miRNAs control cellular proliferation and metastasis by targeting Wnt10b $(12,30)$. This result was consistent with the current finding that transfection with miR-148a mimic suppressed TC cell proliferation and invasion, whereas Wnt10b overexpression reversed this effect. Taken together, the data suggested that this HOTAIRM1/miR-148a/Wnt10b axis controls TC progression. By knocking down HOTAIRM1, it may be possible to inhibit TC cell proliferation and invasion through the miR-148a-mediated suppression of Wnt10b expression.

In conclusion, the present study investigated explored the potential role of lncRNA HOTAIRM1 in inhibiting TC cell proliferation and suppressing the invasive ability by modulating the invasion of TC cells by controlling miR-148a and Wnt10b. This lncRNA mediated the regulation of Wnt10b expression. Taken together, these results suggest that targeting this HOTAIRM1/miR-148a/Wnt10b axis may be represent a potential therapeutic strategy for the treatment of TC.

\section{Acknowledgements}

Not applicable.

\section{Funding}

This study was supported by the National Natural Science Foundation of Jilin Province (grant no. 20180101138JC).

\section{Availability of data and materials}

The datasets used during the present study are available from the corresponding author upon reasonable request.

\section{Authors' contributions}

CL and GC conceived and designed the study. CL, GC, XC and TL performed the experiments. $\mathrm{CL}$ and $\mathrm{GC}$ wrote the manuscript. CL, GC, XC and TL reviewed and edited the manuscript. All authors read and approved the manuscript.

\section{Ethics approval and consent to participate}

The present study conformed to the principles presented in the Declaration of Helsinki and was approved (no. 20190318) by the Ethics Committee of the First Hospital of Jilin University (Jilin, China). Written informed consent was obtained from all study subjects.

\section{Patient consent for publication}

Not applicable.

\section{Competing interests}

The authors declare that they have no competing interests.

\section{References}

1. Miller KD, Siegel RL, Lin CC, Mariotto AB, Kramer JL, Rowland JH, Stein KD, Alteri R and Jemal A: Cancer treatment and survivorship statistics, 2016. CA Cancer J Clin 66: 271-289, 2016.
2. Zhu H, Lv Z, An C, Shi M, Pan W, Zhou L, Yang W and Yang M: Onco-lncRNA HOTAIR and its functional genetic variants in papillary thyroid carcinoma. Sci Rep 6: 31969, 2016.

3. Jendrzejewski J, Thomas A, Liyanarachchi S, Eiterman A, Tomsic J, He H, Radomska HS, Li W, Nagy R, Sworczak K and de la Chapelle A: PTCSC3 is involved in papillary thyroid carcinoma development by modulating S100A4 gene expression. J Clin Endocrinol Metab 100: 1370-1377, 2015.

4. Xing M: Molecular pathogenesis and mechanisms of thyroid cancer. Nat Rev Cancer 13: 184-199, 2013.

5. Sørensen KP, Thomassen M, Tan Q, Bak M, Cold S, Burton M, Larsen MJ and Kruse TA: Long non-coding RNA HOTAIR is an independent prognostic marker of metastasis in estrogen receptor-positive primary breast cancer. Breast Cancer Res Treat 142: 529-536, 2013.

6. Svoboda M, Slyskova J, Schneiderova M, Makovicky P, Bielik L, Levy M, Lipska L, Hemmelova B, Kala Z, Protivankova M, et al: HOTAIR long non-coding RNA is a negative prognostic factor not only in primary tumors, but also in the blood of colorectal cancer patients. Carcinogenesis 35: 1510-1515, 2014.

7. Zhang JX, Han L, Bao ZS, Wang YY, Chen LY, Yan W, Yu SZ, Pu PY, Liu N, et al: HOTAIR, a cell cycle-associated long noncoding RNA and a strong predictor of survival, is preferentially expressed in classical and mesenchymal glioma. Neuro Oncol 15: 1595-1603, 2013.

8. Liu ZM, Wu ZY, Li WH, Wang LQ, Wan JN and Zhong Y: MiR-96-5p promotes the proliferation, invasion and metastasis of papillary thyroid carcinoma through down-regulating CCDC67. Eur Rev Med Pharmacol Sci 23: 3421-3430, 2019.

9. Zhou SL, Tang QL, Zhou SX and Ren RZ: MiR-296-5p suppresses papillary thyroid carcinoma cell growth via targeting PLK1. Eur Rev Med Pharmacol Sci 23: 2084-2091, 2019.

10. Zhang Y, Sun B, Zhao L, Liu Z, Xu Z, Tian Y and Hao C: Up-Regulation of miRNA-148a inhibits proliferation, invasion, and migration while promoting apoptosis of cervical cancer cells by down-regulating RRS1. Biosci Rep 39: BSR20181815, 2019.

11. Sun Y, Zhu Q, Zhou M, Yang W, Shi H, Shan Y, Zhang Q and $\mathrm{Yu} F$ : Restoration of miRNA-148a in pancreatic cancer reduces invasion and metastasis by inhibiting the Wnt/ $\beta$-catenin signaling pathway via downregulating maternally expressed gene-3. Exp Ther Med 17: 639-648, 2019.

12. Shi L, Xi J, Xu X, Peng B and Zhang B: MiR-148a suppressed cell invasion and migration via targeting WNT10b and modulating $\beta$-catenin signaling in cisplatin-resistant colorectal cancer cells. Biomed Pharmacother 109: 902-909, 2019.

13. Shi H, Chen X, Jiang H, Wang X, Yu H, Sun P and Sui X: MiR-148a suppresses cell invasion and migration in gastric cancer by targeting DNA methyltransferase 1. Oncol Lett 15: 4944-4950, 2018.

14. Livak KJ and Schmittgen TD: Analysis of relative gene expression data using real-time quantitative PCR and the 2-(Delta Delta C(T)) method. Methods 25: 402-408, 2001.

15. Choy M, Guo Y, Li H, Wei G, Ye R, Liang W, Xiao H, Li Y and Guan H: Long noncoding RNA LOC100129940-N is upregulated in papillary thyroid cancer and promotes the invasion and progression. Int J Endocrinol 2019: 7043509, 2019.

16. Guo K, Chen L, Wang Y, Qian K, Zheng X, Sun W, Sun T, Wu Y and Wang Z: Long noncoding RNA RP11-547D24.1 regulates proliferation and migration in papillary thyroid carcinoma: Identification and validation of a novel long noncoding RNA through integrated analysis of TCGA database. Cancer Med 1: 3105-3119, 2019.

17. Liang M, Jia J, Chen L, Wei B, Guan Q, Ding Z, Yu J, Pang R and He G: LncRNA MCM3AP-AS1 promotes proliferation and invasion through regulating miR-211-5p/SPARC axis in papillary thyroid cancer. Endocrine 27: 318-326, 2019.

18. Song B, Li R, Zuo Z, Tan J, Liu L, Ding D, Lu Y and Hou D: LncRNA ENST00000539653 acts as an oncogenic factor via MAPK signalling in papillary thyroid cancer. BMC Cancer 19: 297, 2019.

19. Wang XZ, Hang YK, Liu JB, Hou YQ, Wang N and Wang MJ: Over-Expression of microRNA-375 inhibits papillary thyroid carcinoma cell proliferation and induces cell apoptosis by targeting ERBB2. J Pharmacol Sci 130: 78-84, 2016.

20. Liu L, Wang J, Li X, Ma J, Shi C, Zhu H, Xi Q, Zhang J, Zhao X and Gu M: MiR-204-5p suppresses cell proliferation by inhibiting IGFBP5 in papillary thyroid carcinoma. Biochem Biophys Res Commun 457: 621-626, 2015.

21. Li H, Zhao L, Zhang Z, Zhang H, Ding C and Su Z: Roles of microRNA let-7b in papillary thyroid carcinoma by regulating HMGA2. Tumour Biol 39: 101042 8317719274, 2017. 
22. Fang M, Huang W, Wu X, Gao Y, Ou J, Zhang X and Li Y: MiR-141-3p suppresses tumor growth and metastasis in papillary thyroid cancer via targeting yin yang 1 . Anat Rec (Hoboken) 302: 258-268, 2019

23. Leone V, D'Angelo D, Rubio I, de Freitas PM, Federico A, Colamaio M, Pallante P, Medeiros-Neto G and Fusco A: MiR-1 is a tumor suppressor in thyroid carcinogenesis targeting CCND2, CXCR4, and SDF-1alpha. J Clin Endocrinol Metab 96: E1388-E1398, 2011.

24. Han C, Zheng W, Ge M, Wang K, Xiang Y and Wang P. Downregulation of cyclin-dependent kinase 8 by microRNA-148a suppresses proliferation and invasiveness of papillary thyroid carcinomas. Am J Cancer Res 7: 2081-2090, 2017.

25. Xu Y, Han YF, Zhu SJ, Dong JD and Ye B: MiRNA-148a inhibits cell growth of papillary thyroid cancer through STAT3 and PI3K/AKT signaling pathways. Oncol Rep 38: 3085-3093, 2017.

26. Han J, Zhang M, Nie C, Jia J, Wang F, Yu J, Bi W, Liu B, Sheng R, $\mathrm{He} \mathrm{G}$, et al: MiR-215 suppresses papillary thyroid cancer proliferation, migration, and invasion through the AKT/GSK-3 $\beta /$ Snail signaling by targeting ARFGEF1. Cell Death Dis 10: 195, 2019.

27. Chen J, Yin J, Liu J,Zhu RX, Zheng Y and Wang XL: MiR-202-3p functions as a tumor suppressor and reduces cell migration and invasion in papillary thyroid carcinoma. Eur Rev Med Pharmacol Sci 23: 1145-1150, 2019.
28. Xiao Y, Yan X, Yang Y and Ma X: Downregulation of long noncoding RNA HOTAIRM1 variant 1 contributes to osteoarthritis via regulating miR-125b/BMPR2 axis and activating JNK/MAPK/ERK pathway. Biomed Pharmacother 109: 1569-1577, 2019

29. LiQ, Dong C, Cui J, Wang Y and Hong X: Over-Expressed lncRNA HOTAIRM1 promotes tumor growth and invasion through up-regulating HOXA1 and sequestering G9a/EZH2/Dnmts away from the HOXA1 gene in glioblastoma multiforme. J Exp Clin Cancer Res 37: 265, 2018.

30. Peng L, Liu Z, Xiao J, Tu Y, Wan Z, Xiong H, Li Y and Xiao W: MicroRNA-148a suppresses epithelial-mesenchymal transition and invasion of pancreatic cancer cells by targeting wnt $10 \mathrm{~b}$ and inhibiting the Wnt $/ \beta$-catenin signaling pathway. Oncol Rep 38: 301-308, 2017.

This work is licensed under a Creative Commons Attribution-NonCommercial-NoDerivatives 4.0 International (CC BY-NC-ND 4.0) License. 